\title{
Endoscopic ultrasound-guided gastrojejunal anastomosis followed by retrograde colonoscope-assisted metal stenting of the bile duct
}

Over the past decades, the secondary mechanical obstruction of the upper gastrointestinal (GI) tract caused by pancreatic head tumors has been treated with a standard surgical approach. The recent development of minimally invasive techniques like transluminal interventions guided by endoscopic ultrasound (EUS) has opened up new modalities for some patients [1-2].

A 56-year-old woman had been treated with chemotherapy for pancreatic head adenocarcinoma with metastases to the lymph nodes. An endoscopic retrograde cholangiopancreatography (ERCP) with a plastic stent implantation was performed to treat obstructive jaundice. After finishing therapy, the patient presented with symptoms of gastric outlet obstruction. Owing to her poor surgical status, she was referred for endoscopic treatment.

The procedure was begun by advancing a guidewire (Hydra Jagwire, .035 inch, $450 \mathrm{~cm}$; Boston Scientific, Marlborough, Massachusetts, USA) followed by a biliary catheter (X-Press; Olympus Medical Systems Corp., Tokyo, Japan) through the narrowed duodenal bulb to the most distal part of the upper jejunum. This step was performed under fluoroscopic guidance with a standard endoscope (GIFHQ190; Olympus). To exclude a distal small intestinal obstruction, a contrast solution (Omnipaque; GE Healthcare, Chicago, Illinois, USA) was injected behind the Treitz ligament ( $\triangleright$ Video 1 ). After passage confirmation, the small intestine was filled with water at the Treitz ligament level ( $\triangleright$ Fig. 1). This facilitated endosonographic visualization of the optimal site for lumen-apposing metal stent (LAMS) placement. The gastrojejunostomy was performed by implanting a 15-mm stent (AXIOS, Boston Scientific) ( Fig. 2).

After 4 weeks, the patient presented with no symptoms of Gl obstruction but with worsening cholestasis requiring ERCP. Plastic stent removal and self-expandable

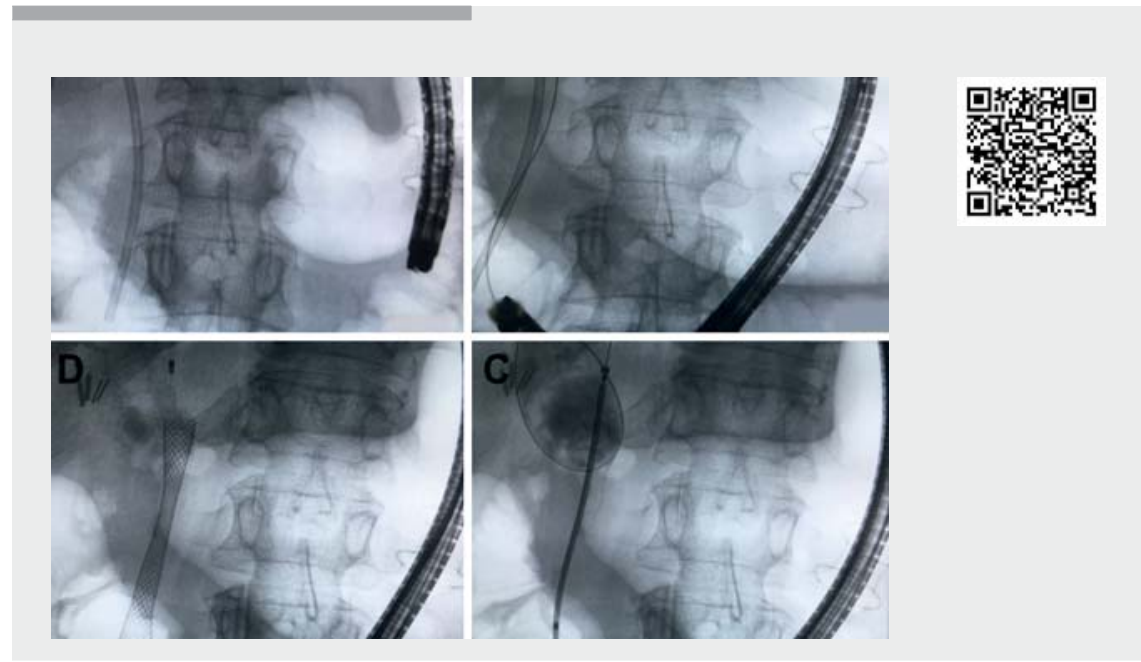

$\checkmark$ Video 1 Sequential endoscopic therapy was performed to create an endoscopic ultrasound-guided gastrojejunal anastomosis, followed by retrograde colonoscope-assisted metal stenting of the bile duct.

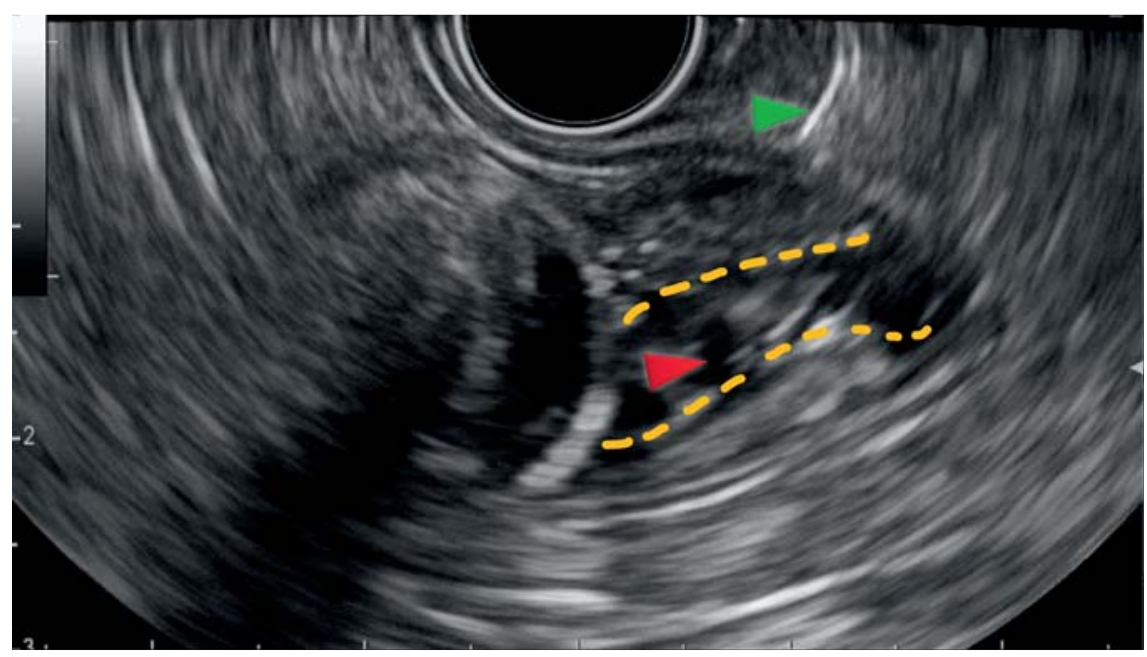

- Fig. 1 The small intestine is filled with water at the Treitz ligament level. The lumen of the small intestine (yellow lines), water in the lumen of the small intestine (red arrow), and the lumen-apposing metal stent before implantation (green arrow) are visible.

metal stent implantation were performed through the AXIOS stent lumen using a pediatric colonoscope (PCF-H190, Olympus) ( $\triangleright$ Video 1, $\triangleright$ Fig.3). At the 4-week follow-up, no jaundice or gastrointestinal obstruction was observed.
This case is important for three main reasons. First, we confirmed that EUS-guided transluminal creation of a gastrojejunal anastomosis is a safe and effective alternative to surgery. From the technical standpoint, we want to highlight the role of the catheter in locating the opti- 


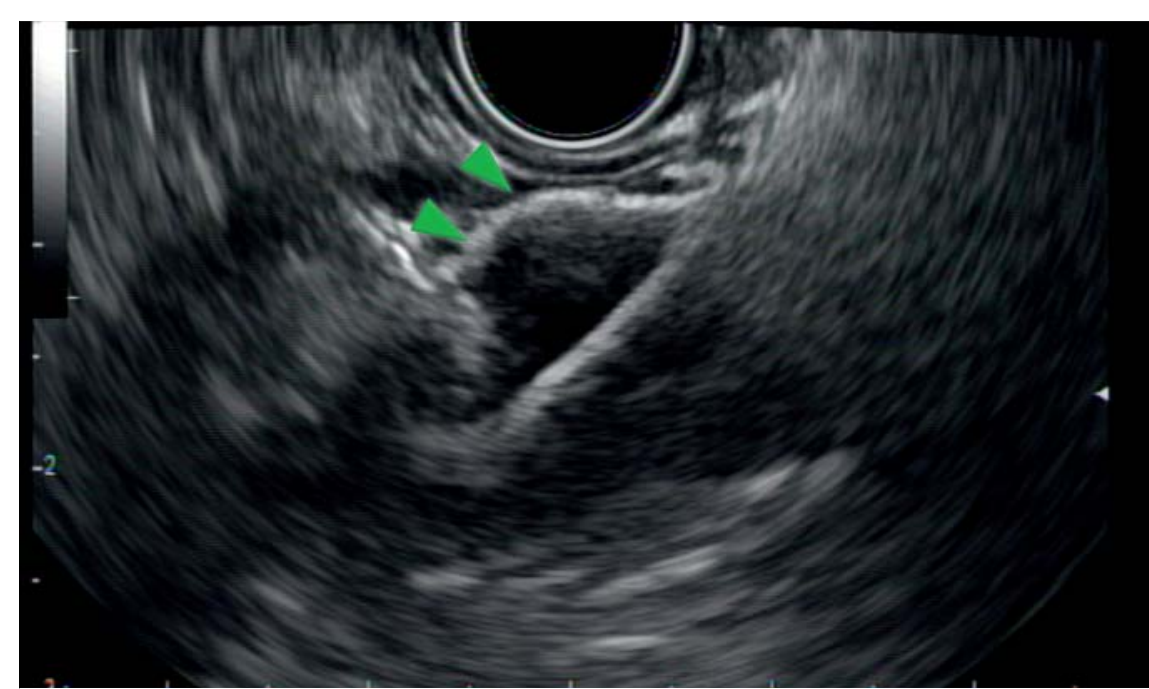

> Fig. 2 The gastrojejunostomy was performed by implanting an AXIOS stent. The internal flank in the lumen of the small intestine (green arrows) is visible.
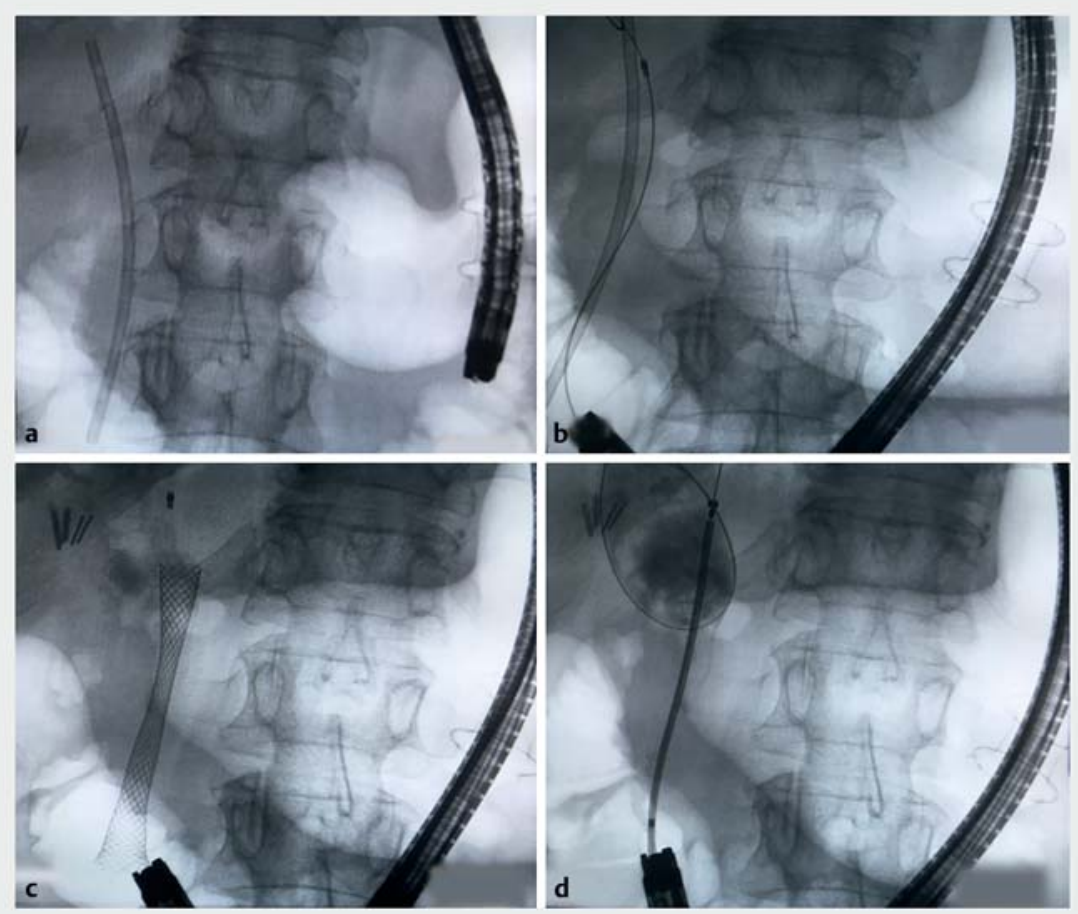

- Fig. 3 a A pediatric colonoscope was advanced through the lumen of the AXIOS stent. b The common bile duct cannulation with visible guidewire. c, $\mathbf{d}$ Implantation of self-expandable metal stent.

mal site for stent placement. Also, filling the jejunum with water is a crucial step for safely performing the gastrojejunostomy because it allows for easy visibility and puncturing during EUS. Second, placement of a self-expandable stent with the relevant diameter facilitates endoscopic gastrojejunostomy, making it in essence a "double retrograde" procedure (“oral retrograde ERCP”).

Endoscopy_UCTN_Code_TTT_1AR_2AK

Competing interests

The authors declare that they have no conflict of interest.

The authors

Artur Raiter ${ }^{1}$, Katarzyna M. Pawlak ${ }^{2}$, Katarzyna Kozłowska-Petriczko ${ }^{3}$, Jan Petriczko ${ }^{4}$, Anna Wiechowska-Kozłowska ${ }^{2}$

1 Department of Endoscopy, Specialist Hospital of Alfred Sokolowski, Wałbrzych, Poland

2 Department of Gastroenterology, Hospital of the Ministry of Internal Affairs in Szczecin, Poland

3 Department of Gastroenterology and Internal Medicine, SPWSZ Hospital, Szczecin, Poland

4 Department of Plastic, Endocrine and General Surgery, Pomeranian Medical University, Szczecin, Poland

Corresponding author

\section{Katarzyna M. Pawlak, MD}

Hospital of the Ministry of Interior and Administration, Department of Gastroenterology, Endoscopy Unit, Jagiellońska 44 st, 70-382 Szczecin, Poland Fax: +48 914329522

pawlakatarzyna@gmail.com

\section{References}

[1] Siddiqui UD, Levy M]. EUS-guided transluminal interventions. Gastroenterology 2018; 154: 1911-1924

[2] Simons-Linares CR, Chahal P. Advances in interventional endoscopic ultrasound (EUS): a technical review. J Clin Gastroenterol 2020; 54: 579-590

Bibliography

performing another required procedure, in this case ERCP. Finally, we have shown that ERCP can be performed using a forward-viewing endoscope (pediatric colonoscope), which was advanced orally but reached the papilla on a retrograde route, i.e., through the newly created
Endoscopy 2021; 53: E315-E316

DOI 10.1055/a-1268-6961

ISSN 0013-726X

published online 19.10.2020

(c) 2020. Thieme. All rights reserved.

Georg Thieme Verlag KG, Rüdigerstraße 14, 70469 Stuttgart, Germany 